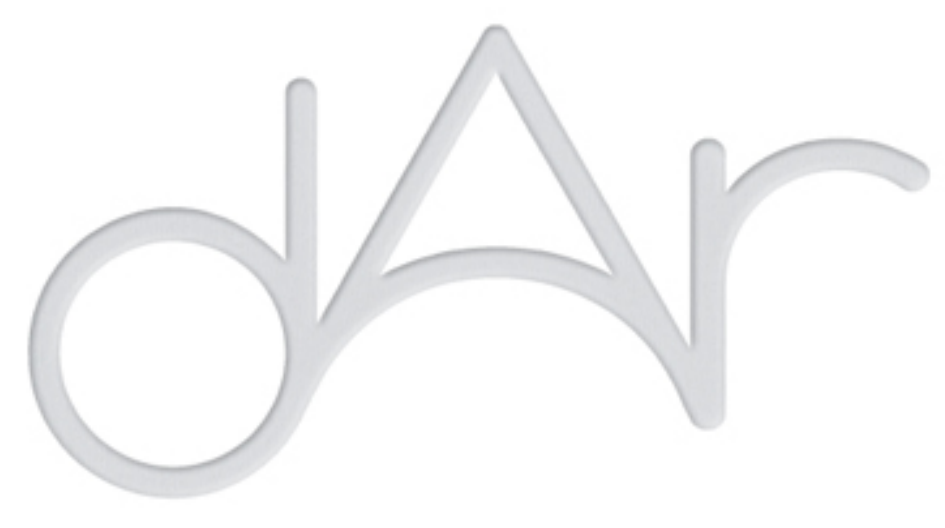

Le savoir faire local et la connaissance des plantes médicinales spontanées dans la région de Tata: etude eco-patrimoniale

Autor(es): $\quad$ Mouhiddine, Mohammed; Belkamel, Abdeljalil; Bammi, Jamal

Publicado por: Imprensa da Universidade de Coimbra

URL

persistente: URI:http://hdl.handle.net/10316.2/44427

DOI: DOI:https://doi.org/10.14195/2182-844X_5_6

Accessed : $\quad$ 26-Apr-2023 14:01:02

A navegação consulta e descarregamento dos títulos inseridos nas Bibliotecas Digitais UC Digitalis, UC Pombalina e UC Impactum, pressupõem a aceitação plena e sem reservas dos Termos e Condições de Uso destas Bibliotecas Digitais, disponíveis em https://digitalis.uc.pt/pt-pt/termos.

Conforme exposto nos referidos Termos e Condições de Uso, o descarregamento de títulos de acesso restrito requer uma licença válida de autorização devendo o utilizador aceder ao(s) documento(s) a partir de um endereço de IP da instituição detentora da supramencionada licença.

Ao utilizador é apenas permitido o descarregamento para uso pessoal, pelo que o emprego do(s) título(s) descarregado(s) para outro fim, designadamente comercial, carece de autorização do respetivo autor ou editor da obra.

Na medida em que todas as obras da UC Digitalis se encontram protegidas pelo Código do Direito de Autor e Direitos Conexos e demais legislação aplicável, toda a cópia, parcial ou total, deste documento, nos casos em que é legalmente admitida, deverá conter ou fazer-se acompanhar por este aviso. 

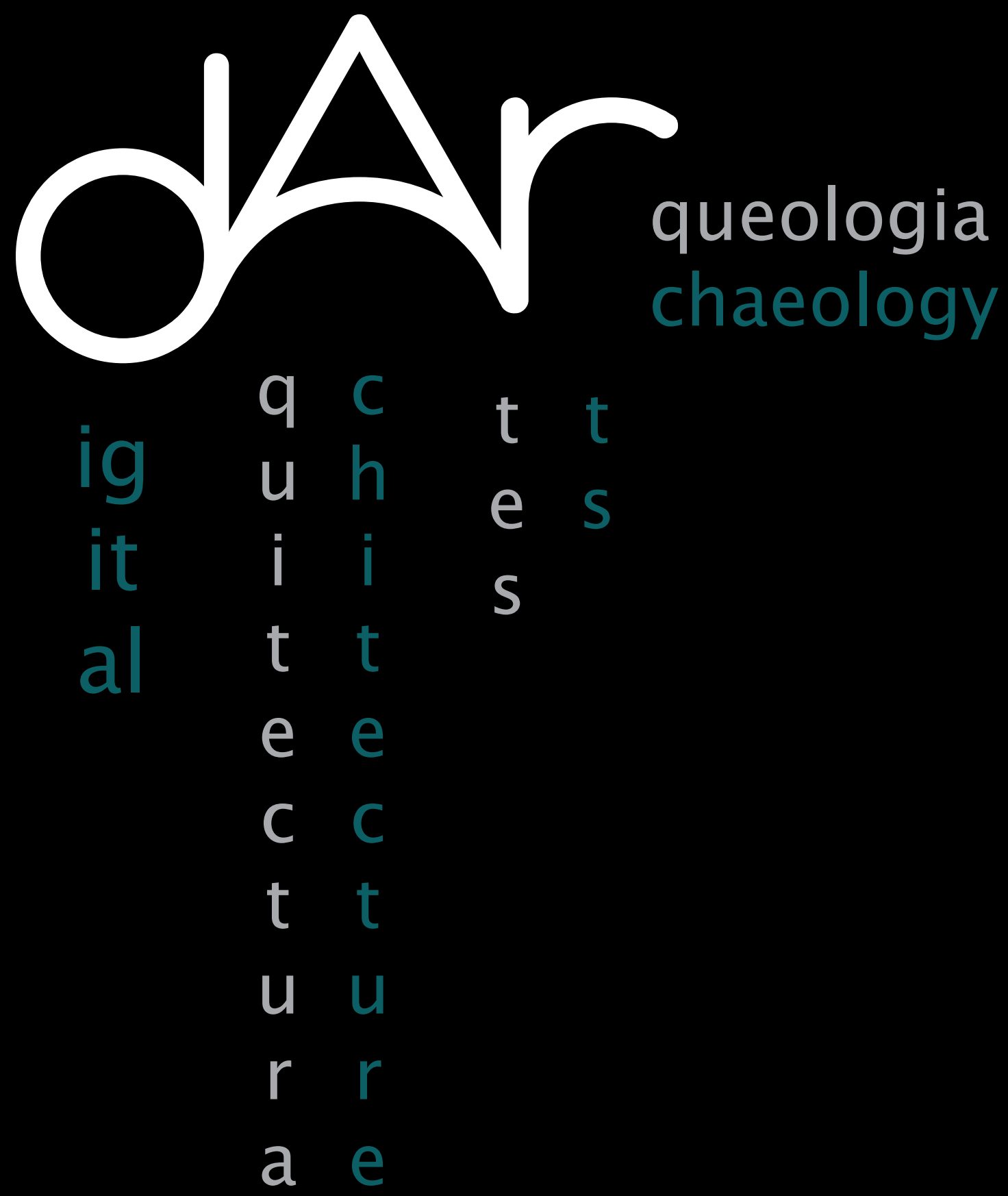

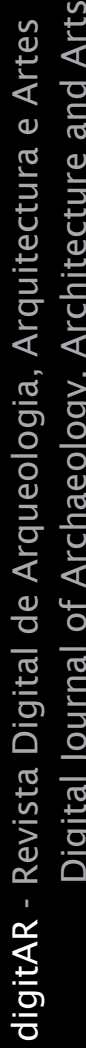




\title{
LE SAVOIR FAIRE LOCAL ET LA CONNAISSANCE DES PLANTES MÉDICINALES SPONTANÉES DANS LA RÉGION DE TATA: ETUdE ECO-PATRIMONIALE
}

\author{
Dr. Mohammed Mouhiddine, F.LS.H Ben M'sik. Casablanca \\ Dr. Abdeljalil Belkamel, FST, Marrakech \\ Dr. Jamal Bammi, Faculté des Sciences Kénitra
}

\section{Résumé}

Une étude ethnobotanique a été réalisée dans les oasis de la Province de Tata afin d'identifier les différents modes d'utilisation et d'exploitation des plantes médicinales spontanées.

Des enquêtes réalisées durant l'année 2008/2009 ont permis d'inventorier 45 espèces médicinales et aromatiques.

Cette étude se veut une contribution à la connaissance du savoir faire local dans des écosystèmes oasiens en matière de soins naturels tout en mettant l'accent sur l'importance de ce capital, de point de vue scientifique et social, et la nécessité de le sauvegarder pour une potentielle mise en valeur.

Mots-clés: Plantes médicinales, ethnobotanique, oasis, Tata, Draa, Maroc.

\section{Introduction}

Partout dans le monde, la médecine, en tant que manifestation du génie et du savoir-faire d'un groupe humain, c'est d'abord un fait de culture, un fait social. Ceci dit, il nous a semblé qu'en restituant aux choses le cadre naturel de leur mouvance, nous leur restituerons en même temps la profondeur historique et la densité culturelle qu'elles n'ont, dans tout les cas, jamais perdue tout à fait, et que, de ce fait, nous rendrions plus facile la préhension de notre objet, à travers trois des grandes interactions gouvernant le monde des hommes et des choses : l'homme- le milieu, l'espace- la durée, l'actuel- le passé (Bellakhdar, 1978).

Comparée à d'autres régions du Maroc, celle de la région de Tata présente des handicaps sur certains points notamment la disponibilité en eau et l'éloignement géographique.

https://doi.org/10.14195/2182-844X_5_6 
En effet, les territoires oasiens à Tata connaissent de fortes pressions environnementales: raréfaction de la ressource en eau, désertification, et érosion de la biodiversité. Malheureusement, la société oasienne nomade, semi-nomade et sédentaire est très touchée par la pauvreté dont les répercussions se manifestent surtout par des conflits liés au droit foncier et aux usages de l'eau. Aujourd'hui on y constate un abandon progressif des activités agricoles et de nouvelles pratiques sociales en rupture avec les règles antérieures de gestion du milieu.

Dans ces conditions, la stratégie de développement à adopter est de valoriser ce que l'on appelle les avantages locaux. En effet, par sa localisation, son contexte géographique, topographique et social, mais aussi grâce aux savoirs et savoir-faire locaux, cette région du sud du Maroc présente des avantages pour diverses particularités régionales.

Les connaissances empiriques, en matière de soins traditionnels se sont transmises verbalement à travers les générations et se sont enrichies grâce à une situation géographique stratégique entre l'Afrique du nord, le Sahara et le Sahel. Cet enrichissement est aussi lié aux événements historiques et au brassage des civilisations amazighes, juives, sahariennes, et arabomusulmanes au niveau de ces oasis (Bellakhdar et al., 1992). Cet héritage, comme pour d'autres régions au Maroc, a comme ossature le savoir amazighe et arabo-musulman ayant suscité depuis longtemps la curiosité d'ethnobotanistes marocains et étrangers (El Rhaffari et Zaïd, 2002).

$C^{\prime}$ est ce qui a été à l'origine de l'hypothèse sur laquelle est basée notre étude : les plantes médicinales et aromatiques peuvent représenter un atout pour le développement local ? Plusieurs éléments plaident pour ce point de vue :

- Premièrement, ces plantes sont présentes de façon très diversifiée dans cette région.

- Deuxièmement, on assiste de plus en plus à un regain d'intérêt pour la médecine traditionnelle au Maroc et les pharmacopées régionales, en plus les marchés des produits naturels biologiques au niveau international sont en plein essor (Steer et al. 2004).

Par ailleurs, l'origine de l'exploitation de des ressources naturelles, notamment les plantes médicinales, remonte à des temps reculés. Mais il ne $s^{\prime}$ agit pas pour autant uniquement de tradition car ces plantes sont toujours intensément utilisées par les populations locales. En effet, la totalité des gens que nous avons interrogés utilisent les plantes médicinales et une grande partie d'entre eux récoltent eux-mêmes des plantes.

Notre travail est articulé autour de patrimoine oasien selon les pratiques de soins traditionnels à base de plantes dans les Oasis de la province du Tata. 
C'est à travers une étude ethnobotanique que nous rapportons ici les résultats de l'enquête qui a mis en exergue l'utilisation de 45 plantes médicinales et aromatiques dans un contexte patrimonial en relation avec savoir faire local à la fois synchronique et diachronique.

\section{L'importance de l'oasis est dans son contexte patrimonial}

Le patrimoine oasien est le fruit d'un territoire créé par l'Homme, ce générateur est situé en milieu aride et semi-aride, dont l'aménagement n'est possible que par la présence d'eau souterraine, donc les oasis sont un patrimoine culturel néoformé par l'homme d'une façon progressive à travers l'histoire, malgré la pénurie des biens de la nature.

Les précipitations annuelles sont respectivement inférieures à $250 \mathrm{~mm}$ par an avec une évapotranspiration extrêmement importante. L'oasis rompt avec l'aridité environnante grâce à la création d'un espace de verdure, telle que la palmeraie. L'espace de verdure, du fait de sa conception et de son fonctionnement, génère un climat local plus frais et humide qui permet le développement d'une biodiversité endémique et atypique. L'agriculteur, jardinier du désert et gardien de l'héritage oasien, à un rôle primordial dans la durabilité des systèmes oasiens et dans leurs richesses en biodiversité.

Le territoire oasien connaît de fortes pressions environnementales: raréfaction de la ressource en eau, désertification, et érosion de la biodiversité.

Plusieurs mesures globales et intégrées feraient en sorte d'épargner aux oasis de Tata et ses environs un sort qu'ont imposé la sécheresse, le Bayoud (le redoutable champignon du palmier dattier), l'épuisement de la nappe phréatique et les politiques isolées et sectorielles.

La problématique oasienne exige ainsi des interventions ciblées et efficientes. $\mathrm{Au}$ centre de toutes ces actions prévues, l'homme devrait requérir une importance capitale.

\section{Présentation de la zone d'étude}

La zone d'étude correspond à la province de Tata, zone présaharienne du sud du Maroc, qui s'étend du versant sud de l'Anti-Atlas à l'oued Draa qui marque la frontière avec l'Algérie et les régions sahariennes. Plus exactement, la province s'enserre dans un vaste couloir continental Est-Ouest constitué de glacis et de terrasses alluviales, bordé au nord par le Jbel Bani et au sud par l'oued Draa, pour s'ouvrir sur le terminal anti-atlasique à travers des Foums.

Les foums, des fissures régulières dans cette chaîne montagneuse étroite, 
constituent des points de collecte d'eau, ce qui a rendu possible l'agriculture irriguée dans cette zone aride. Il s'agit habituellement d'oasis de taille moyenne, dont Foum El Hassane, Akka, Tata, Agadir-Tissint et Foum Zguid constituent les plus grands périmètres irrigués. Les données suivantes sont basées sur une étude menée en 1993 et 1994 par De Haas (1995).

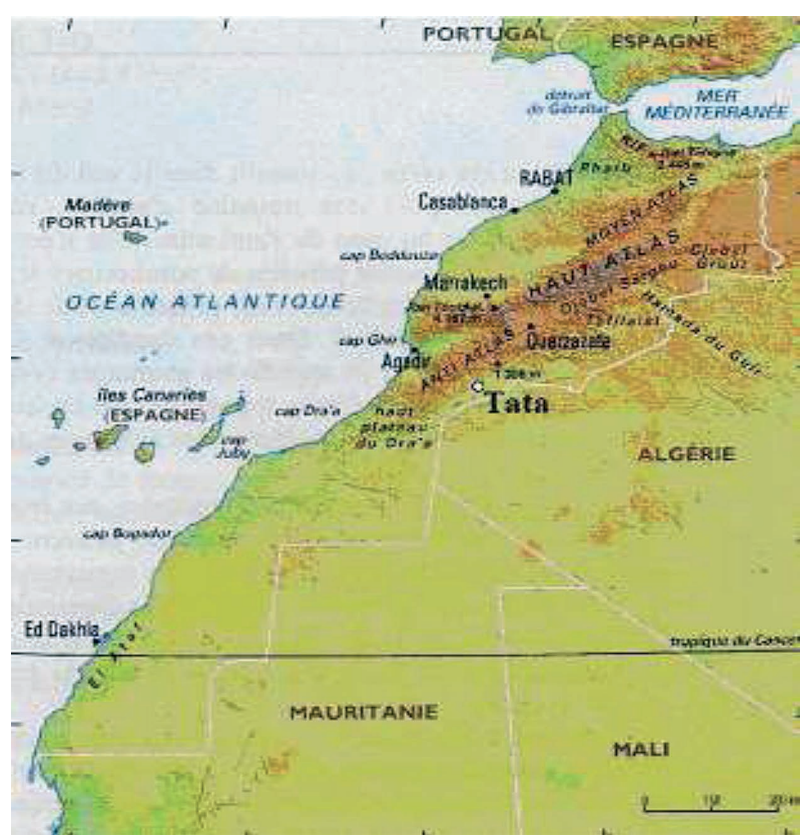

Fig. 1 - Localisation géographique de la Province de Tata

La faiblesse des précipitations est révélatrice de l'aridité du climat. En effet, l'ensemble des sites d'enquêtes (Foum Zguid, Tata, Akka, Tissint) reçoivent moins de $100 \mathrm{~mm}$ de pluies annuelles. De plus, il existe une variabilité inter-annuelle importante des précipitations qui fait véritablement de la gestion de la ressource en eau une composante centrale des oasis (Steer et al. 2004). Le climat aride est de type saharien et les températures maximales peuvent dépasser les $50^{\circ} \mathrm{C}$. La végétation visible hors oasis est assez clairsemée.

Seuls les espaces oasiens, grâce à la mobilisation d'eau, présentent une végétation abondante. De plus, la variabilité climatique interannuelle est très grande. Elle entraîne des périodes de sécheresse qui durent parfois 2 à 3 ans. Ainsi, même au sein des oasis, l'eau est une ressource rare et convoitée. Les zones non irrigables contrastent avec les oasis vertes et très influencés par l'action anthropique. Chaque oasis est généralement centrée sur une palmeraie (Steer et al. 2004).

\section{Données socioculturelles}

L'émigration a causé un alourdissement des taches agricoles des femmes. Outre leurs taches domestiques très lourdes, elles sont également responsables pour une grande partie des travaux agricoles, comme le 
désherbage, l'entretien quotidien des palmiers, la récolte et surtout l'élevage. Grâce à la contribution pesante des femmes, la persistance de la société et de l'économie oasienne est assurée.

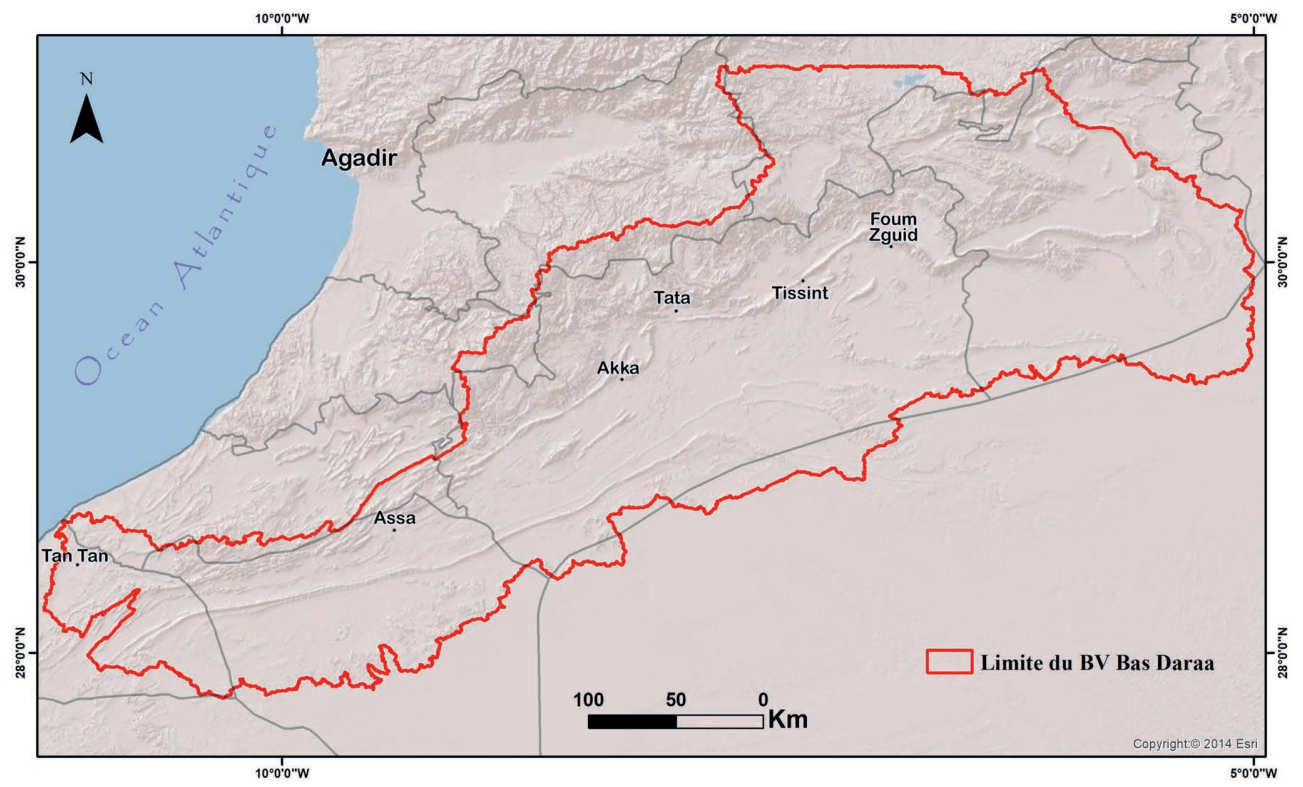

Fig. 2 - Limites de province de Tata dans le Bas Draa

Il va sans dire que l'émigration a profondément bouleversé la société oasienne, sur le plan économique mais aussi sur le plan culturel et psychosocial. L'émigration est devenue un élément intégré et indispensable du genre de vie des oasiens. Pour les jeunes hommes, la question n'est pas de savoir s'ils émigrent, mais plutôt de trouver l'opportunité et le moment propice. Ainsi la migration est souvent caractérisée comme un «rite de passage» (Massey et al. 1993). Il est question d'un vrai «bouleversement mental», qui a également abouti à une désaffection pour l'agriculture (De Haas et De Mas, 1997). Toutes ces raisons incitent à diversifier les approches de développement de cette région du Maroc à travers des angles de visions multiples notamment ceux liés au développement durable par l'exploitation rationnelle des ressources naturelles. C'est justement dans cette optique que s'insère notre vision qui se veut une contribution à la valorisation des plantes médicinales et aromatiques de la région. Nous nous contentons, dans un premier temps de fournir des données botaniques et ethnologiques qui serviront de base pour d'autres études en relation directe avec des projets éventuels de mise en valeur. 


\section{Histoire des Plantes Médicinales dans La Province de Tata : l'exemple de l'Oasis de Tissint}

L'Oasis de Tissint eût autrefois un passé glorieux. Située sur la route menant de Sijilmassa (près de l'actuelle Rissani) au Souss, en un endroit propice aux palmeraies et à l'agriculture en raison de l'abondance relative de l'eau, il représentait un lieu de passage et d'approvisionnement incontournable pour tous ceux qui, pasteurs, marchants migrants- traversaient le Sahara marocain d'Est en Ouest.

Cette prospérité dura jusqu'à 1850, période à laquelle Tissint commence à perdre sa prospérité. De sorte qu'en 1884, date de passage de Foucault dans la région, l'oasis n'était déjà plus ce qu'il avait été autrefois et, sur le plan commercial du moins, ne se distinguait plus des autres bourgades de la région. Sur le plan agricole, Tissint demeurait encore une palmeraie productive et bien entretenue (Bellakhdar et al., 1987).

Tissint était jusqu'aux années soixante, un grand marché de plantes médicinales ; les droguistes d'Algérie, du Niger, du Sénégal, de l’Egypte, de Libye et de bien d'autres contées africaines venaient y puiser de grandes quantités de plantes médicinales (Akhmisse, 2004).

L'activité d'herboristerie des habitants de Tissint serait née au départ de l'activité générale d'échange des oasiens du Draa. Celle-ci fut certainement renforcée et diversifiée grâce aux connaissances botaniques des nomades Ida-ou-Blal qui gravitait autour de Tissint, habitués, afin d'assurer leur subsistance, au ramassage de produits alimentaires ou médicinaux (Bellakhdar et al. 1987).

L'activité d'herboristerie semble plus spécialement centrée au ksar Zaouïa. Les herboristes de Tissint sont en effet principalement des serviteurs du Marabout Sidi Abdallah Ou M'Hand en qui ils reconnaissent le saint patron de la profession et en l'honneur duquel se tient chaque année un grand Moussem. A cette date tous les herboristes en déplacement dans le Nord du Maroc doivent être rentrés à Tissint, à défaut de quoi, dit la tradition locale, leur art et leurs affaires déclineraient (Bellakhdar et al. 1987).

Tout incite à penser que le saint patron de Tissint aurait pu avoir une activité de médecin/ apothicaire qui avait acquis une connaissance approfondie des plantes régionales ainsi que leurs vertus médicinales et avait-il transmis son savoir botanique et médical à sa descendance et ses serviteurs, ceux-là même qui aujourd'hui pratiquent le métier.

Ce savoir ancestral se transmet de génération en génération. Mais la spécialisation dans cette activité est aussi la conséquence de l'organisation 
sociale du village. En effet, ces herboristes n'ont pas d'accès à la terre et à l'eau et ils ne peuvent donc pas avoir de revenus par l'agriculture. Dans ce contexte, l'herboristerie contribue amplement à assurer les besoins vitaux des familles de l'oasis.

Sur les cinq quartiers que compte la village de Tissint, un est spécialisé dans cette activité : sur les 260 familles de ce quartier, 200 pratiquent l'herboristerie. Ces herboristes ne se contentent pas de cueillir et de vendre les plantes médicinales, ce sont en fait de véritables tradipraticiens (Steer et al. 2004).

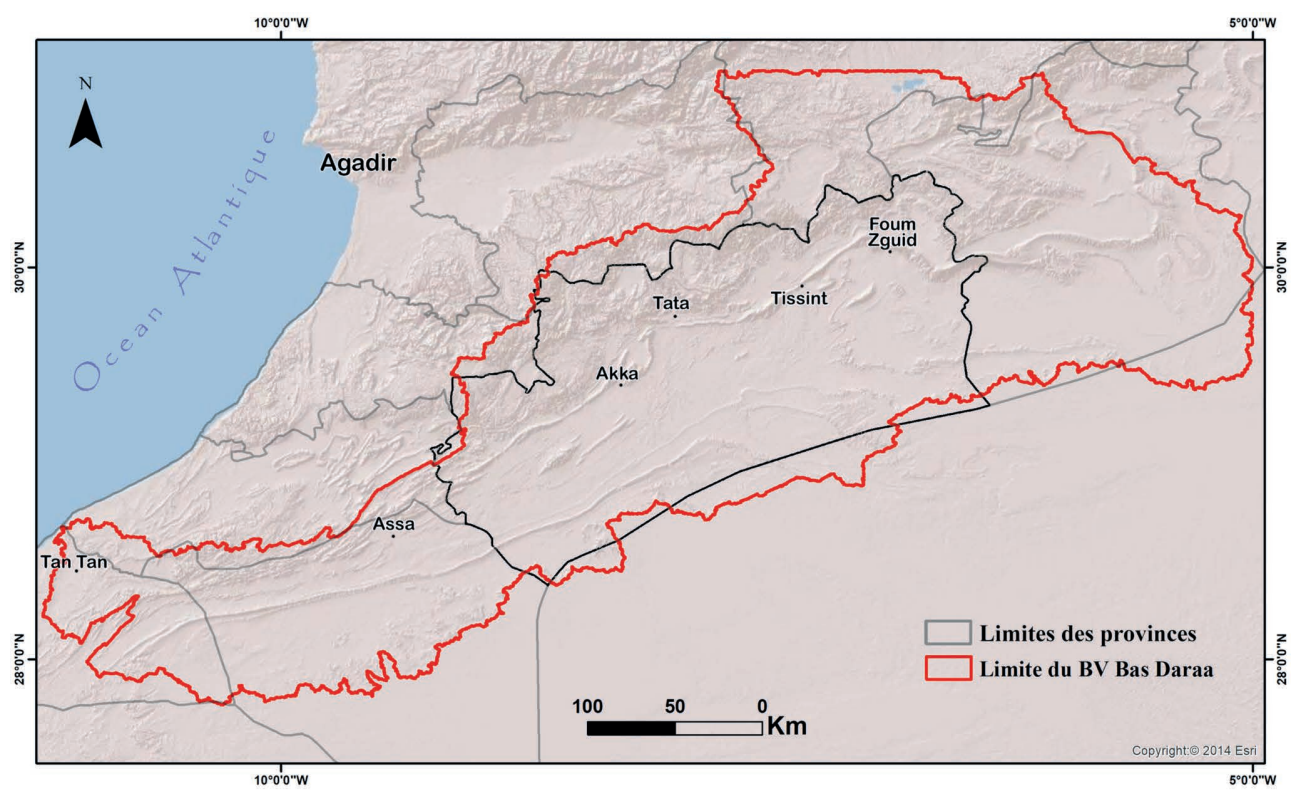

Fig.3 : Zones d'enquêtes dans la Province de Tata

\section{Démarche et méthodologie}

Les données d'exploration du terrain, nous renseignent sur la localisation et la validation de l'aire de répartition des formes et des espèces de la région d'étude. Le choix des techniques à mettre en œuvre, tient compte de la Géographie, la Géomorphologie, la Géologie, la Biologie et de l'Ecologie des êtres vivants.

Les plantes médicinales et aromatiques n'ont, jusqu'à présent, été que peu étudiées dans la région. En effet, notre étude se veut une contribution à mettre en évidence la diversité des plantes spontanées utilisées dans les 
soins traditionnels.

Les éléments d'enquêtes rapportés dans le présent travail ont été réunis à la suite des visites dans les oasis de la province de Tata. Le premier travail de terrain a eu lieu en Octobre-novembre 2008 et le second en Mars 2009, puis suivit par d'autres visites printanières.

Nous avons choisi nos sites d'enquêtes dans les importants oasis de la région : Foum Zguid, Tissint, Tagmout, Aqqa et Tata (Fig.2). Par la suite, nous avons identifié sur le terrain les différentes espèces végétales signalées par les populations locales comme ayant des vertus médicinales.

Une attention particulière a été accordée à l'Oasis de Tissint qui revêt un intérêt incomparable en matière de plantes médicinales et aromatiques. $C^{\prime}$ est la raison pour laquelle, la majeure partie des enquêtes ethnobotaniques a été réalisée aux près des herboristes de ce oasis.

Les résultats sont présentés sous forme d'un catalogue où sont rapportées les informations suivantes :

- Le nom scientifique de la plante médicinale.

- La famille botanique (F.B)

- Le non vernaculaire français disponible (N.V).

- Le nom amazigh (N. Amz)

- L'utilisation thérapeutique traditionnelle de la plante (U.T).

La vérification systématique des taxons a été réalisée en utilisant les documents spécialisés notamment Quezel et Santa (1963) et Ozenda (2004 [1983]).

\section{Résultats et Discussion}

6. 1. Catalogue des Plantes Médicinales Spontanées des Oasis de la Province de Tata.

Acacia radiana Savi.

FB : Mimosacées ; N.V : acacia ; N. Amz : Amrad; U.T : la gomme (Tiffizit) est très prisée contre la fatigue générale. Utilisée aussi contre l'asthme. Les racines en poudre sont stomachiques.

Aizoon canariense L.

FB : Aizoacées ; N.V : l'aizoon des Canaries: N.Amz : Taghassoult. U.T. en période de sécheresse, les graines noires réduites en poudre étaient fortement consommées. 
Ammodaucaus leucothrichus Coss et Dur.

FB : Apiacées; N.V : cumin velu; N.Amz :lkammoun; U.T: les graines en poudre sont stomachiques, contre les coliques des enfants. Indiqué contre les maladies respiratoires et comme hypotenseur. Aromatique culinaire.

Anastatica hierochuntica L.

FB : Brassicacées ; N.V : Anastatica; N. Amz: takchmat/lkachtam; U.T : plante réduite en poudre contre les refroidissements. Plante très puisée par les femmes contre la stérilité. L'infusion contre les maladies oculaires.

Anvillea radiata Coss et Dr.

FB : Astéracées ; N.V : / ; N. Amaz : Wajjrg ; U.T : les graines en poudre, seules ou en mélange avec de l'huile d'olive s'utilisent contre les refroidissements.

Artemisia inculta Del.

FB : Astéracées; N.V: armoise; N.Amz: Izri; U.T. plante en décoction est vermifuge et stomachique. Considérée aussi comme antidiabétique.

Asphodelus fistulosis L.

FB: Liliacées; N.V: asphodèle; N. Amz : Taziyit; U.T: les graines en poudre sont anti-hémorrö̈daires, les feuilles fraîches en cataplasme sont antirhumatismales.

Bubonium odorum (Schousb.) Maire.

FB: Astéracées ; N.V: /; N. Amz: taougout ; U.T. poudre de feuilles contre la migraine, l'infusion de la plante, en gargarisme contre les douleurs dentaires.

Calotropis procera Will.

FB : Asclépiadacées; NV : pomme de Sodome; N.Amz: Tawrja. U.T. très connue dans la région par sa toxicité. En application externe, le latex est très efficace contre les verrues et durillons. 
Capparis spinosa L.

FB: Capparidacées; NV: Câprier; N.Amz: Tayloloute; U.T. Le fruit séché, pulvérisé est utilisé dans les refroidissements, les rhumatismes et la stérilité féminine. En usage externe, les boutons floraux sont utilisés en emplâtre contre la chute des cheveux.

\section{Caylusea hexagyna Forsk.Green}

FB: Résédacées; N.V : ? ; N. Amz : Azeldar/Timimt; U.T: la décoction des feuilles utilisée par les femmes pour fortifier les cheveux.

Centaurea pungens Pomel.

FB: Astéracées; N.V : centaurée ; N. Amz: neggayr; U.T: plante très puisée contre les refroidissements.

Chrysanthemum trifurcatum Desf.

FB : Astéracées; N.V: chrysanthème; N. Amz: tayrright; U.T: la plante en infusion contre les maladies hépatobiliaires. C'est un aromate de thé des nomades.

Citrullus colocynthis (L.) Schrad.

FB: Cucurbitacées ; NV: Coloquinte ; N.Amz: Aferziz/Taferzizt; U.T: Le fruit chauffé coupé en deux est mis sous le pied pendant une heure pour combattre le rhume. Il est appliqué sur les piqûres de scorpion. Antidiabétique à dose infinitésimale.

Cleome arabica $\mathrm{L}$.

FB: Capparidacées ; N.V : cléone; N.Amaz: Lkhanza. U.T : les gousses réduites en poudre sont antitussives. Utilisée en cataplasme comme fébrifuge.

Cotula cinerea (Del.) Vis.

FB: Astéracées; N.V : / ; N. Amz: tiklilt; U.T: fébrifuge, stomachique. Utilisée comme aromate du thé. 


\section{Cressa cretica L.}

FB: Convolvulacées; N.V: /; N. Amz: wammach ; U.T : utilisée en friction contre les morsures de scorpions et serpents.

Euphorbia calyptrata Coss et Dr.

FB: Euphorbiacées ; N.V : euphorbe; N. Amz: rmada ; U.T : la poudre de la plante est utilisée comme laxatif.

Fagonia cretica L.

FB: Zygophyllacées; N.V: /; N. Amz : Tliha; U.T: les feuilles en cataplasme sur les blessures sont vulnéraires.

Farsetia aegyptiaca Turra.

FB: Brassicacées; N.V: /; N. Amz: tassit; U.T: l'infusion de la plante en gargarisme contre les douleurs dentaires et gingivales.

Forskahlea tenacissima L.

FB: Urticacées; N. : /; N. Amz: Lessig; U.T: la tige feuillée en infusion contre les maladies hépatobiliaires.

Gaillonia reboudiana Coss. et Dur.

FB: Rubiacées; N.V:/; N. Amz: Tibskit ntmghart ; U.T: très prisée par les femmes de la région après l'accouchement. Les feuilles utilisées contre les refroidissements.

Haloxylon scoparium Pomel

FB: Chénopodiacées; N.V: haloxylon; N.Amz: assay; U.T: les racines sont utilisées contre les troubles gastriques. L'infusion de feuilles sert comme collyre contre les inflammations oculaires. La poudre de feuilles est antidiabétique.

Haplophyllum vermiculare Hand et Mazz. 
FB: Rutacées ; N.V: /; N. Amz: Awermi/ Tiwraghin; U.T: la plante en infusion est stomachique, diurétique et contre les refroidissements. Toxique à forte dose.

Heliotropium bacciferum Forsk.

FB: Borraginacées; N.V héliotrope: N.Amz: Taynost. U.T. très consommée par les nomades avant floraison. Réduite en poudre et appliquée en cataplasme, elle serait résolutive des abcès et furoncles.

Launea arborescenns (Batt.) Maire.

FB: Astéracées, N.V: /; N. Amz: lferskel; U.T: latex utilisé comme résolutif contre les abcès et furoncles. Egalement pour sevrer les bébés.

Maerua crassifolia Forsk.

FB: Capparidacées, N.V : Méruée; N.Amaz: atil; U.T. La plante utilisée en décoction, contre les maladies gastriques et hépatiques.

Marrubium deserti De Noë

FB: Lamiacées; N.V : marrube du désert; N. Am : Jaâyda ; U.T : tiges feuillés très puisés contre les refroidissements.

Marrubium vulgare L.

FB: Lamiacées; N.V: marrube blanc ; N. Amz: Ifzi; U.T : expectorante, béchique, anti-ictérique, antidiabétique.

Mesembryanthemum nodiflorum $\mathrm{L}$.

FB: Aizoacées. NV griffe du diable: N.Amz: Aqbarou ; U.T. à l'état sec, la plante est réduite en poudre ou en infusion sert à combattre les intoxications.

Morettia canescens Boiss.

FB: Brassicacées ; N.V:/; N. Amz: tabzwalt; U.T : les feuilles en décoction utilisées comme lotion pour fortifier les cheveux.

Nitraria retusa Forsk.

FB: Zygophyllacées; N.V: /; N. Amz: Agrzim U.T: les feuilles fraîches appliqués sur les boutons sont maturatives. Les feuilles en décoction sont anti- 
hémorroïdaires.

Opuntia ficus indica (L.) Mill.

FB: Cactacées; N.V : opuntia; N.Amaz: Taknourit; U.T: infusion des fleurs contre la diarrhée.

Panicum turgidum Forsk.

FB: Poacées ; N.V: panicum; N. Amz: tigoucine; U.T : la décoction de la tige souterraine est utilisée contre les maladies respiratoires notamment l'asthme.

Peganum harmala L.

FB: Zygophyllacées; N.V : Harmel; N. Amz: Lharmel; U.T: les graines sont antirhumatismales et anti-hémorroïdaires, en usage externe, la décoction des graines est fortifiante des cheveux. Toxique à forte dose.

Pergularia tomentosa L.

FB: Asclépiadacées. N.V pergularia: N.Amz: Lghalga. U.T. par application externe, le latex est utilisé comme maturatif des abcès et furoncles. Il est en outre très employé pour extirper les épines de la peau. Plante toxique.

Plantago coronopus L.

FB: Plantaginacées; N.V: plantain à cornes; N. Amz:Talma; U.T: feuilles utilisées comme diurétiques. Les graines sont anti-diarrhéiques.

Reseda villosa Coss.

FB: Résédacées; N.V: réséda; N. Amz: Irgjidi ; U.T: les graines sont utilisées comme aphrodisiaques.

Retama retam Webb.

FB: Fabacées; N.V: rétame; N. Amz: Allouco; U.T: les feuilles et fleurs sont appliquées sur les blessures comme vulnéraire.

Spergularia marginata Kittel

FB: Caryophyllacées; N.V : spergularia ; N.Amaz : boughlam. U.T : la racine est considérée comme antirhumatismale et aphrodisiaque. La poudre de racine s'utilise contre la stérilité féminine. 
Tamarix gallica L.

FB: Tamaricacées; N.V: tamarix; N. Amz;: U.T : la poudre de feuilles est vermifuge. En infusion les feuilles sont anti-rhumatismales.

Warionia saharae Benth et Coss.

FB: Astéracées; NV: Warione du sahara; N. Amz: Alliijjane ; U.T. Les feuilles en décoction son utilisées contre l'ictère. Les racines et les feuilles en décoction sont utilisées comme bain de bouche contre les maux des dents et les aphtes. Les feuilles en décoction contre les douleurs rhumatismales.

Withania adpressa Coss.

FB: Solanacées; N.V:/; N. Amz : Aglim ; U.T: la poudre des feuilles est utilisée contre les intoxications.

Ziziphus lotus (L.) Desf.

FB: Rhamnacées; N.V : jujubier; N. Amz: Azaggar; U.T: l'infusion des fruits est fébrifuge, les feuilles en cataplasme mûrissent les abcès.

Zygophyllum gaetulum Emb. et Maire

FB: Zygophyllacées; N. : Zygophyllum; N. Amz: l'aggaya; U.T: la poudre des feuilles est utilisée comme vulnéraire. Très prisée dans la région de Foum Zguid contre les maladies gastriques. Réputée aussi antidiabétique.

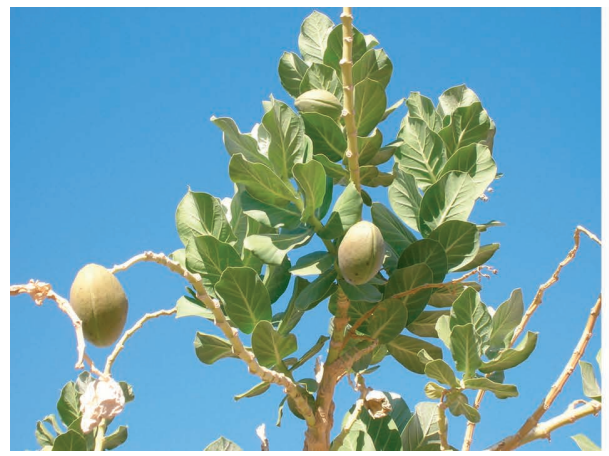

Photo 1 - Calotropis procera (photo. Jamal Bammi)

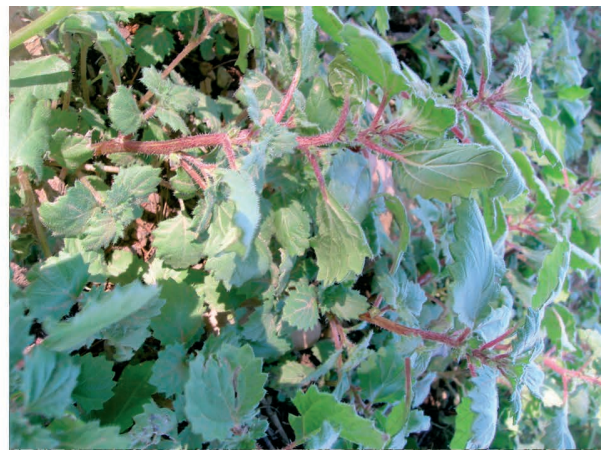

Photo 2. Forskahlea tenacissima (photo. Jamal Bammi) 


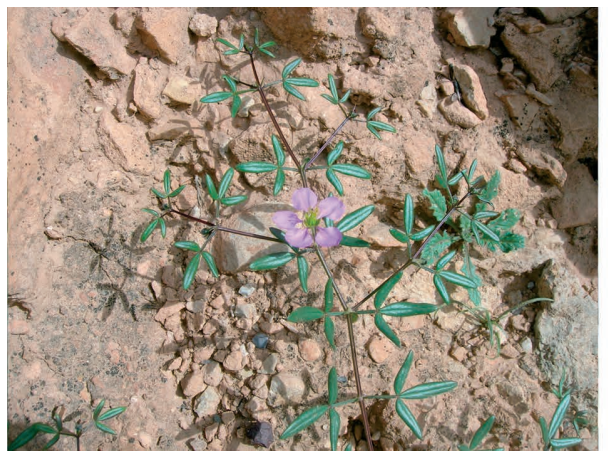

Photo 3. Fagonia cretica (photo. Jamal Bammi)

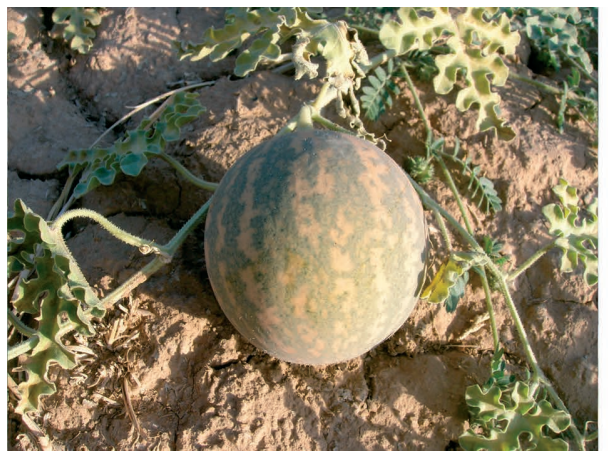

Photo 5. Citrullus colocynthis (photo. Jamal Bammi)

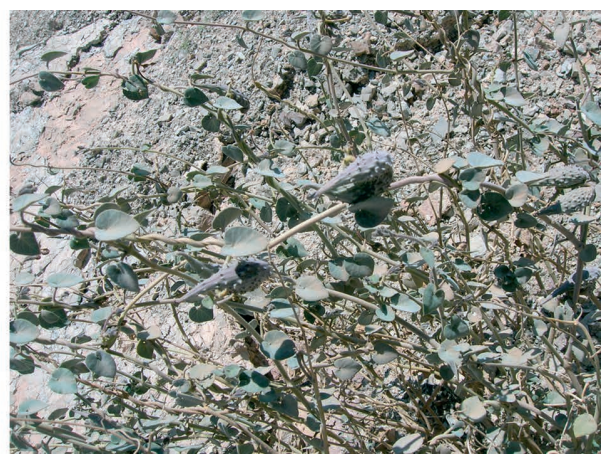

Photo 4. Pergularia tomentosa (photo. Jamal Bammi)

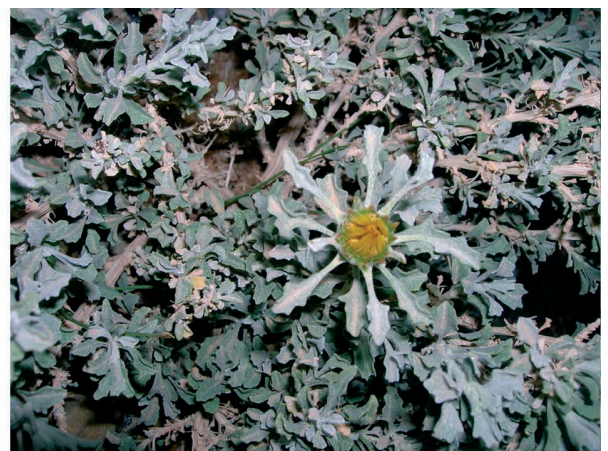

Photo. 6 Anvillea radiata (photo. Jamal Bammi)

\section{2. Discussion}

De point de vue soins traditionnels, nos résultats concordent avec d'autres études ethnobotaniques menées et effectuées dans les différentes régions du Sud Marocain et au Sahara (Bellakhdar, 1978 ; Bellakhdar et al. 1987 ; Maiza et al. 1993 ; El Rhaffari et Zaid, 2002). Un point original caractérise notre étude qui est l'intérêt donné uniquement aux plantes spontanées. C'est, à notre sens, le moyen méthodologique adéquat pour insérer le savoir anthropologique local dans son contexte écosystémique.

Mais il existe en général peu de valorisation des plantes médicinales : après la récolte, celles-ci sont mises à sécher et utilisées telles quelles pour des infusions. Elles ne font l'objet d'aucun conditionnement. 
Il y a très peu de commercialisation, sauf dans le cas de Tissint. En effet, dans ce village Oasis la vente des plantes médicinales représente le principal moyen de subsistance de toute une partie de la population. Les herboristes de Tissint se déplacent dans tout le Maroc pour vendre leurs plantes mais chacun a son propre circuit de commercialisation. Il y a deux principaux modes de vente de ces plantes : sur les souks (principalement ceux des grandes villes) et sur commande. En effet, ces herboristes ont un réseau de clients bien établi qui les appellent pour obtenir les plantes dont ils ont besoin. Ainsi, les hommes des familles d'herboristes voyagent tout au long de l'année. Ils ne rentrent à Tissint que pour assister au Moussem De Sidi Abdallah Om'hand (Bellakhdar et al., 1987).. Ils profitent de ce retour pour se fournir en plantes que leur femme et leurs enfants ont récoltées pendant toute l'année.

Bellakhdar et al. (1987), ont noté que les professionnels de l'herboristerie à Tissint sont classés en trois catégories : au haut de l'échelle ceux qui font les taymust c'est-à-dire les secrets de certaines mixtures et formes galéniques complexes. En raison de leur savoir, ils sont l'objet d'une grande considération de la part de leurs confrères herboristes et de l'ensemble de la population de la région ; viennent ensuite les herboristes qui -sans posséder l'art des précédents- savent quand même préparer quelques mélanges généralement "lamsakhen" c'està-dire les réchauffants. Enfin, il y a la majorité des herboristes qui ne sont que des négociants en plantes médicinales.

Par ailleurs, le secteur des plantes médicinales est fortement lié à des savoirs que les gens ne souhaitent pas partager. Il existe beaucoup de « secrets » autour de cette activité. Il en résulte que le savoir ancestral en matière de pharmacopée traditionnelle est en péril étant donné que la première catégorie d'herboristes qui détient le savoir original ne le transmet ni aux confrères des deux autres catégories ni aux générations futurs. C'est là, une problématique qui reste à creuser...

$\mathrm{Au}$ niveau des compétences des herboristes, il n'existe pas une, mais une multitude de connaissances autour des plantes. De l'avis même des herboristes, tous n'ont pas le même niveau de savoir et certains ont des spécialités qu'ils ne veulent pas partager. Mais cette multitude de savoirs concerne aussi les matières premières utilisées. Certains herboristes ont acquis une connaissance fine du milieu et des plantes qui représente pour eux un avantage sur les autres herboristes. Ainsi un grand nombre d'entre eux est opposé à toute organisation de la filière de peur que ces secrets soient divulgués aux autres herboristes. 
Il faut enfin noter que même les circuits de commercialisation sont différents d'un herboriste à l'autre. Une mise en commun dans la filière pourrait conduire certains herboristes à perdre des marchés qu'ils sont pour l'instant les seuls à occuper. Par le passé, il y a déjà eu une tentative d'organisation des herboristes mais celle ci a échoué car ils considéraient que leurs intérêts étaient trop divergents.

Dansce contexte, on peut se demander si une organisation professionnelle de la filière est vraiment envisageable. Or ceci représente un réel handicap pour l'évolution et le développement du secteur (Steer et al, 2004).

Par ailleurs, nous consacrerons la prochaine étude sur le savoir médical oasien aux aspects phytochimiques et pharmacodynamiques, pour mieux cerner le potentiel local, en matière de plantes médicinales et aromatiques, et proposer les modes d'exploitations convenables.

\section{Conclusion}

Les plantes médicinales dans la région de Tata sont jusqu'à présent mal étudiées. Aussi les références bibliographiques sur le sujet sont rares. Les résultats que nous avons exposés constitueraient un socle sur lequel vont venir se greffer d'autres travaux en vue de la mise en valeur de ce secteur digne d'intérêt. Dans une perspective de développement, il semble nécessaire d'approfondir certaines des connaissances que nous avons acquises. C'est notamment le cas pour les vertus des plantes médicinales et le conditionnement de certains produits locaux. Il semble, d'après les résultats auxquels nous sommes parvenus, qu'il existe plusieurs plantes médicinales de haute valeur thérapeutique confirmée par des études scientifiques rigoureuses.

Il en ressort d'ailleurs une spécialisation locale, due soit au contexte biophysique et climatique, soit aux savoir-faire local. Nous avons vu en particulier que la cueillette systématique des plantes médicinales se fait uniquement à Tissint. Il s'impose d'emblée que le développement durable doit valoriser les potentialités locales et diversifier les ressources et les activités de la région entière.

\section{Références bibliographiques}

Akhmisse M. 2004. Le journal d'un médecin chez les berbères de Bani. Dar Kortoba, Casablanca. 139 p. 
Bellakhdar J. 1978. Médecine traditionnelle et toxicologie ouest-sahariennes. Contribution à l'étude de la pharmacopée marocaine. Editions Techniques Nord Africaines. Rabat. 357p.

Bellakhdar J., Baayaoui A., Kazdari A. et Maréchal J. 1987. Herboristes et médecine traditionnelle à Tissint, oasis présaharien du sud marocain (Province de Tata). AL Biruniya. Rev.Mar. Pharm. Tome 3, n 1. p 7-50.

Bellakhdar J., Benabid A., Vittoz J. et Maréchal J.1992. Tissint, Une Oasis Du Maroc Présaharien (Monographie D’une Palmeraie Du Moyen Dra). Ed. Al Biruniya, Rabat.243 p.

De Foucauld (1888). Reconnaissance au Maroc (1883-1884). Paris, A. Chalamel, réédition 1985. Paris: Éditions d'Aujourd'hui.

De Haas H. et De Mas P., 1997. Retombées Ecologiques et Humaines de la Migration dans l'Agriculture Marginale des Oasis et Montagnes Marocaines. L'Émigration Maghrébine vers 1'Europe: Espace et Investissement. Cahiers du CEMMM, no. 5. Oujda: Université Mohammed Ier, p 47-74.

De Haas, H. (1995). Développement socio-économique et agricole dans une oasis présaharienne marocaine: Agadir-Tissint, Province de Tata. Thèse de Troisième Cycle. Amsterdam: Institut de Géographie Humaine, Université d'Amsterdam. In De Haas H. et De Mas P., 1997.

EL Rhaffari L., Zaid A. 2002. Pratique de la phytothérapie dans le sud-est du Maroc (Tafilalet). Un savoir empirique pour une pharmacopée rénovée. Dans : Des sources du savoir aux médicaments du futur. 4ème congrès européen d'ethnopharmacologie Fleurentin, J., J.-M. Pelt and G. Mazars (Eds.). Paris, IRD Editions. p 293-318.

Ghani Zakaria, jamal Bammi et Mohamed Mouhiddine, 2009. Sainteté et écologie au Maroc : un essai anthropologique ,in RGM, N 1-2, Rabat, Vol 25, pp 197- 212

Maiza K., Brac De La Perrière. R .A., Hammiche V. 1993. Pharmacopée traditionnelle saharienne : Sahara septentrional. Actes du $2^{\text {ème Colloque }}$ Européen d'Ethnopharmacologie et de la 11» Conférence internationale d'Ethnomédecine, Heidelberg-, 24 -27 mars.

Massey D., J. Arango, G. Hugo, A. Kouaouci, A. Pellegrino \& J. Taylor 1993. Theories of International Migration: A Review and Appraisal. In: Population and Development Review. 19, No.3.

Mouhiddine M., 2013. Protection et valorisation du patrimoine naturel et culturel du Bas Draa, in Patrimoine culturel matériel dans la région SoussMassa-Draâ, IRCAM-Rabat, PP : 307-323,

Ozenda P. 2004. Flore et végétation du Sahara. CNRS éditions p 662.

Quézel, P. et Santa S. 1962. Nouvelle flore de l'Algérie et des régions désertiques 
méridionales. CNRS., Paris, 2 tomes, 1170 pages.

Steer L., Goudet M., Akchour M., Mouradi Abdellah H.,. 2004. Les plantes aromatiques, médicinales et tinctoriales, un atout pour le développement rural de la région de Tata. Centre National d'Etudes Agronomiques des Régions Chaudes. En collaboration avec DPA (Tata) et ALCESDAM (Maroc). Edition : P. JOUVE, C. SEUGE. France. 45 p. 